\title{
EFFICACY OF INTRA-ARTICULAR LIQUID PHASE CONCENTRATED GROWTH FACTOR INJECTION IN TREATMENT OF DISC DISPLACE- MENT WITH REDUCTION: A CLINICAL STUDY
}

\author{
Mohammad Mostafa Zaabal ${ }^{1}$, Khalid Mohammad Ali² , Bahaa El-din Abd-Rabbo Tewfik ${ }^{3}$
}

\begin{abstract}
Objectives: to evaluate the efficiency of intra-articular CGF injection in comparison with conventional arthrocentesis in the treatment of TMD. Subjects and Methods: Twenty patients suffering from TMD were included in the study diagnosed according to The Research Diagnostic Criteria for Temporomandibular disorders (RDC/TMD) and divided into two groups as follow; ten patients underwent intra-articular injections of $2 \mathrm{ml} \mathrm{CGF}$ into the temporomandibular joint (TMJ) (group I) and the other ten patients were treated by the conventional arthrocentesis (group II). The patients were clinically evaluated preoperatively and postoperatively at the intervals of 1 month, 3 , and 6 months. Results: The differences between the two groups in all the measured parameters were statistically insignificant throughout the postoperative period. But CGF group showed superior result than arthrocentesis group regarding pain, mouth opening and inferior result in clicking. Conclusion: CGF injection is a safe and effective method in the treatment of TMDs.
\end{abstract}

KEY WORDS: TMJ, TMDs, CGF, Arthrocentesis.

\section{INTRODUCTION}

The temporomandibular joint (TMJ) is a compound joint that can be classified by anatomic type as well as by function. TMJ is composed of the temporal bone and the mandible, as well as a specialized dense fibrous structure, the articular disk, several ligaments and numerous associated muscles ${ }^{(1)}$.

Temporomandibular joint disorders (TMDs) are a form of musculoskeletal pain of the TMJ and masticatory muscles of nonspecific etiology $y^{(2)}$.
A number of conservative methods are used in the treatment of TMDs including occlusal splints, supportive physical therapy procedures, rehabilitation involving muscular training, and psychological support $^{(3)}$. Intra-articular administration of medications is an established method of treatment, particularly in orthopedic and rheumatic disorders associated with pain, effusion, inflammation of cartilage, bone and joint capsules as well as fibrous adhesions. Currently, agents used for intra-articular injection within the temporomandibular joint region include hyaluronic acid and steroids ${ }^{(4)}$.

1. Dentist at Ministry of Health, Masters Candidate, Oral and Maxillofacial Surgery Dept., Faculty of Dental Medicine, Al-Azhar University (Cairo- boys).

2. Professor of Oral and Maxillofacial Surgery, Faculty of Dental Medicine, Al-Azhar University (Cairo- boys).

3. Assistant Professor of Oral and Maxillofacial Surgery, Faculty of Dental Medicine, Al-Azhar University (Cairo- boys).

-Corresponding author: mohammedzabaal@gmail.com 
Growth Factors (GFs) is a naturally substance capable of stimulating cellular growth, proliferation, migration, healing and cellular differentiation. Usually it is a protein or a steroid hormone. GFs are secreted by all cellular components of the skin such as keratinocytes, melanocytes, fibroblasts, adipose tissue and by alpha granules of blood platelet ${ }^{(5)}$.

Many researchers referred the high efficacy of platelet-rich plasma (PRP) in the management of musculo-articular disorders and its safety to the use of autologous material and it has an anabolic effect on chondrocytes, mesenchymal stem cell and synoviocytes with resultant increases in cell proliferation, cartilaginous extracellular matrix accumulation, and increase hyaluronic acid secretion as well as the low costs of treatment ${ }^{(6)}$. PRP has the potential to inhibit inflammation and alleviate osteoarthritis symptoms with a clinically acceptable safety profile ${ }^{(7)}$.

Concentrated Growth Factor (CGF) is another modified form of PRP, are prepared by repeatedly switching the centrifugation speed and are characterized as a relatively stiffer fibrin clot. Therefore, it has been anticipated that the difference in mechanical characteristics may produce a difference in the growth factor content ${ }^{(8)}$. This study was designed to evaluate the clinical efficacy of CGF in the treatment of TMD.

\section{SUBJECTS AND METHODS}

Twenty patients with anterior disc displacement with reduction were included in this study. The patients were selected among those attending outpatient clinic of Oral and Maxillofacial Surgery, AlSayed Galal University Hospital, Cairo, Al-Azhar University. Each patient signed an informed written consent having all details about the procedure. The diagnosis was made on the basis of Research Diagnostic Criteria for Temporomandibular Disorders (RDC/TMD). The ethical clearance was obtained by the ethical committee before the study began and the selected patients were informed about the nature of the study and informed consents were signed.
Patients were divided into two groups: Ten patients were treated by intra-articular liquid Phase Concentrated Growth Factor injection (LPCGF) in TMJ (Group I); and ten patients were treated by standard arthrocentesis (Group II).

Patients were included if their age ranged from 18 to 50 years, and who have unilateral or bilateral disc displacement with reduction.

Patients were excluded if they had uncontrolled systemic disease, hematological, neurological, inflammatory or connective tissue disorder. Patients who had a history of maxillofacial trauma, previous TMJ surgery; head or neck cancer; or using anticoagulant drugs. Pregnant women, and patients with unilateral or bilateral disc displacement without reduction were also excluded.

I. Preoperative evaluation: Clinical evaluation: all details were recorded in a questionnaire by the examiner including chief complaint, personal data, past history, general body examination, evaluation of measurement of maximum mouth opening, lateral movements, protrusive movement, presence of joint sounds, dental evaluation, masticatory muscles tenderness. Complete blood picture was done to ensure the counts of platelets were within normal limits.

Radiographic evaluation: Panoramic radiography was obtained for screening to rule out any severe condylar changes. MRI was obtained to confirm the initial diagnosis of anterior disc displacement with reduction.

II. Operative procedure: In both groups, under general anaesthia (GA), disinfection by Povidone-iodine and A tragus-lateral canthus line from the tragus to the lateral canthus was drawn corresponding to the method of Murakami ${ }^{(9)}$.

Group I: LPCGF was prepared according to Sacco protocol ${ }^{(10)}$. Ten milliliters of blood were drawn from the patient's vein, and the blood was transferred into plastic cap tubes without 
anticoagulants and reagents. $30 \mathrm{sec}$ acceleration, 2 min 2700rpm, 4 min 2400rpm, 4 min 2700rpm, $3 \mathrm{~min} 3000 \mathrm{rpm}$, and $36 \mathrm{sec}$ deceleration and stop. At the end of the process, there were 3 blood fractions: the upper layer was represented by Platelet- poor plasma; the middle layer was represented by a very large and dense LPCGF and the lower layer was red blood cells. Then, the middle layer (LPCGF) was separated. Using two 22- gauge needles at points $\mathrm{D}$ and $\mathrm{E}$. Then, 2 $\mathrm{mL}$ of physiological saline was injected, and the smoothness of inflow and outflow was observed to ensure that the position of the injection was within the superior joint space. Next, the needle was removed from point $\mathrm{E}$ before injecting the extracted $2 \mathrm{~mL}$ of LPCGF at point $\mathrm{D}$.

Group II: 22 Gauge needle was inserted into the superior joint space at the glenoid fossa. Approximately $2 \mathrm{ml}$ of Ringer's lactate solution* (Ringer's lactate, Egypt Otsuka Company, Cairo, Egypt) was then injected to distend the superior joint space. A second 22-guage needle was inserted into the distended compartment in the area of articular eminence to establish a free flow of the solution through the superior joint space. This needle provides an outflow for the solution. A total of $100 \mathrm{ml}$ of solution was used to lavage the superior joint space, during which time the outlet needle was momentarily blocked with finger pressure 2 or 3 times to help distend and break up the joint adhesions. Once the needles were removed, the patient's jaw was gently manipulated in the vertical, protrusive and lateral excursions.

III. The Post-Operative Phase: Oral soft diet for 3 days and gradually resume to normal diet after 3 days, post-operative analgesic Paracetamol $500 \mathrm{mg}$ tablet, one tablet every $8 \mathrm{hrs}$ for 3 days and Amoxicillin/clavulanate potassium $1 \mathrm{~g}$ tablet, one tablet every $12 \mathrm{hrs}$ for 5 days to protect against any possibility of infection.

IV. Follow-up Phase: Follow up was done at 1 month, 3 months and 6 months intervals. Postoperative evaluation includes the following parameters: Pain level using Visual analogue scale (VAS) from zero to ten. Maximum inter-incisal opening measured by the distance in millimeters between the incisal edges of the upper and lower central incisors in vertical direction at maximum pain free mouth opening by using caliper.

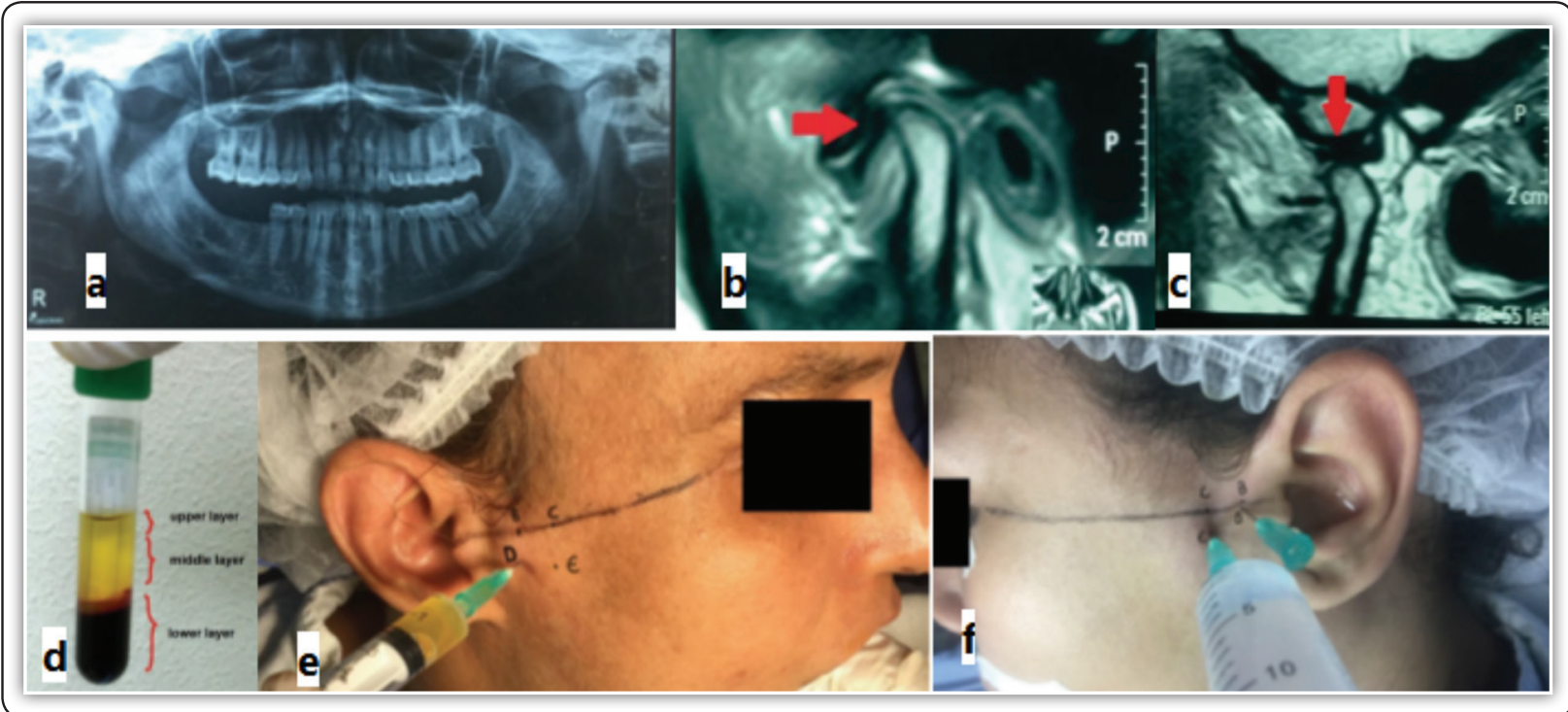

FIG (1) a; Panoramic view. b; MRI image illustrated anterior disc displacement in closed. c; MRI illustrated disc recapture in open position. d; plastic tube containing 3 blood layers. e; landmarks Canthal-tragus line and points of injection during injection of CGF in superior joint space. f;Arthrocentesis procedure 
Range of lateral and protrusive movements measured by the distance in millimeters between the upper and lower midlines in horizontal direction on lateral and protrusive movements by using caliper. Presence of joint noise at opening and closing was evaluated by light bilateral digital palpation during jaw movement. Presence or absence of joint tenderness on palpation: recorded as tender or not tender.

\section{Statistical analysis of the data:}

The data was collected and statically analyzed using SPSS (Statistical Package for Social Sciences) version 20 INC US.

\section{RESULTS}

The present study has been conducted on twenty patients (18 females and 2 males) selected from those attending the outpatient clinic of the Oral and maxillofacial Surgery Department, Al-said Galal Hospital, Al-azhar University. Their age ranged from 18 to 50 years with a mean of $33.52 \pm 7.81$.

Patients were divided into two groups each consisting of ten patients. All patients in group I were females. In group II, they were eight females and two males. All patients evaluated by clinical diagnosis that was made on the basis of DC/TMD questionnaire axis I survey. All patients in both groups were followed up for 6 months.

No major adverse reaction related to CGF injections or arthrocentesis were observed during injection and follow-up period. In group I, patients presented with mild pain and swelling at the site of the injection for 2 days after injection which resolved spontaneously without any intervention. In group II, mild swelling and tenderness lasted for one week and also it resolved spontaneously. There were no sign of infection or restriction of the joint movement for both groups.

The statistical analysis of pain intensity data showed significant VAS scores at 1 month, 3 months, and 6 months. The VAS value was decreased in both groups throughout the follow-up periods but there was more improvement in group I than group II, in Group I was statistical significant difference $(p<0.05)$., and else statistical significant difference $(\mathrm{P}<0.05)$ in Group II.

Regarding the maximum mouth opening, there was a preoperative limited mouth opening but the mouth opening improved postoperatively. The maximum mouth opening scores at 1 month, 3 months, and 6 months. The mouth opening increased in both groups throughout the follow-up periods, but in Group I was statistical significant difference $(p<0.05)$, and statistical significant difference $(\mathrm{P}<0.05)$ in Group II.

The protrusive movement increased in both groups throughout the follow-up periods, but no statistical significant difference $(\mathrm{P}>0.05)$. Regarding the lateral movement was measured in groups I \& II. They were increased in group I \& group II throughout the follow up periods, but the difference between the two groups throughout the whole follow-up period was found to be statistically insignificant.

Regarding to TMJ clicking in (group I) eight of the ten patients $(80 \%)$ had detectable joint sounds, this decreased to three patients out of ten $(30 \%)$ by the end of the 6 months' follow-up period. There was significant difference after 3 month and 6 month follow up period in this group.

In (group II) nine of the ten patients (90\%) had detectable joint sounds. By the end of the 6 months follow-up period, four patients $(40 \%)$ were still complaining of clicking. There was a significant difference when comparing preoperative values with 6 month ( $\mathrm{p}=0.019)$. Comparing the two groups, There was no statistically significant difference between the two groups regarding TMJ clicking preoperatively and during the postoperative follow up period ( $\mathrm{p}>0.05)$. 
Table (1): Comparison between the two groups according to VAS, mouth opening, lateral movement, protrusive movement.

\begin{tabular}{|c|c|c|c|c|c|c|}
\hline & \multicolumn{2}{|c|}{ Group 1} & \multirow{2}{*}{ P-Value } & \multicolumn{2}{|c|}{ Group 2} & \multirow{2}{*}{ P-Value } \\
\hline & Mean & \pm SD & & Mean & \pm SD & \\
\hline \multicolumn{7}{|l|}{ VAS } \\
\hline Preop. & 7.563 & 0.928 & & 7.041 & 0.909 & \\
\hline Month 6 & 0.901 & 0.633 & $<0.001$ & 2.262 & 0.617 & $<0.001$ \\
\hline \multicolumn{7}{|c|}{ Mouth opening } \\
\hline Preop. & 31.878 & \pm 2.829 & & 29.594 & \pm 2.935 & \\
\hline Month 6 & 41.247 & \pm 3.097 & $<0.001$ & 34.822 & \pm 3.527 & 0.002 \\
\hline \multicolumn{7}{|c|}{ Lateral Movement } \\
\hline Preop. & 9.086 & 1.219 & & 8.449 & 1.005 & \\
\hline Month 6 & 9.36 & 1.325 & 0.636 & 8.661 & 0.945 & 0.633 \\
\hline \multicolumn{7}{|c|}{ Protrusive Movement } \\
\hline Preop. & 8.627 & 1.565 & & 7.239 & 1.255 & \\
\hline Month 6 & 9.059 & 1.578 & 0.546 & 7.4 & 1.275 & 0.779 \\
\hline
\end{tabular}

$P$-value: between preoperative values and postoperative outcomes

Statistically significant at $p \leq 0.05$

\section{DISCUSSION}

TMDs pain is the most frequently occurring, non-dental, chronic pain condition in the orofacial area $^{(11)}$. In recent years, it has been clearly demonstrated that TMDs allow extracellular matrix, collagen, and other excessive metabolic reactions to change the microenvironment around the TMJ so that the cartilage degenerates and the subchondral bone is damaged. Aside from the displacement of the disc position, pain and TMJ dysfunction are also related to the pressure in joint and the cytokine level in synovial fluid. By injecting CGF into the joint, not only the volume of the joint cavity expand so that the internal pressure in the joint can be modulated, but growth factors are synthesized through the alpha granule in degranulation plaques ${ }^{(12)}$.

In a comparative study that has been done between PRP and CGF which explained that CGF preparations contained significant amounts of platelet counts, concentrations of growth factors (TGF- $\beta 1$, PDGF-BB, VEGF), concentrations of the pro-inflammatory cytokines (IL-1 $\beta$, IL-6), WBCs and exert distinguishable actions on periosteal cell proliferation, more potent in angiogenesis and subsequent wound healing which give rise to osteoblasts involved in periodontal skeletal regeneration ${ }^{(13)}$.

The growth factors can restore the disc, capsule, retro-discal pad and achieve efficacy in inhibiting pro-inflammatory cytokines through inhibiting interleukin-1 released by the activated macrophages and can help regenerate new bone, fibrocartilage, hyaline cartilage and better ultrastructural architecture of the collagen fibrils ${ }^{(14)}$.

In the present study eighteen patients were females \& two patients were males with age range varied from 18 to 50 years with a mean age range of $33.52 \pm 7.81$. This in agreement with many studies 
which concluded that women had a significantly higher prevalence of clinical signs.

The pain reduction in group I is in agreement with the results obtained by several authors. Pain decrease after injection of CGF was shown to be related to: early release of protease activated receptor 4 peptides from alpha granule in the platelets which has analgesic and anti-inflammatory effect lead to early relief of pain. The pain reduction in group II in agreement with the results obtained by several authors who reported an improvement in the pain level post arthrocentesis in their studies by washing out of inflammatory mediators by arthrocentesis which had its effect in pain reduction and increasing range of movement but the improvement is highly significance in group I than group II ${ }^{(15)}$.

The improvement in mouth opening movement in group I was coincide with the results obtained by several authors (16) who reported an increase in the measurements of mandibular movements including maximal inter-incisal opening, lateral and protrusive movements in their studies after injection of CGF and follow up for 12 months has been done . Nitzan et al ${ }^{(17)}$ reported that arthrocentesis proved to be highly effective in providing significant improvement in maximal mouth opening and lateral movement toward the unaffected side in all patients. They claimed that physiotherapy following such treatment produced further improvement which comes in accordance with the results of group II. In addition, many authors reported the improvement of masticatory efficiency following arthrocentesis. But the improvement in group I is highly significance than group II ( $4.02 \pm 2.54$ in group I and $1.34 \pm 2.14$ in group II, $\mathrm{P}<.01)$.

However, many studies showed improvement of clicking after one injection of CGF patients in this study showed less improvement as in the study of Moon et al. ${ }^{(18)}$ who found CGF derivatives was effective in the treatment of TMJ pain and restricted mouth opening but it was not effective in the treatment of clicking. These findings of group II goes in line with the results obtained by Nitzan et al. (17) who reported that thirteen of seventeen patients did not have clicking after arthrocentesis. It was also in agreement with Yoda et al. ${ }^{(19)}$ who found that nine of the twelve joints showed improvement in clicking after arthrocentesis.

In the present study, when comparing the clinical outcomes to the preoperative values, both groups showed improvement in all the measured parameters except lateral, protrusive movement and clicking in two groups that showed less improvement, when comparing between the two groups outcomes, it was found to be non-significant. Thus demonstrating the effectiveness of both methods in treatment of TMD patients.

Unfortunately, this study was not free from limitation which was related to postoperative evaluation of MRI images and this occurred due to its cost, limited availability and lack of awareness or uncooperative patient. And also follow up period 12 months was considered to evaluate long term changes in the outcome.

\section{CONCLUSION}

CGF injection was simple, minimally invasive procedure and easy to obtain from the patient. CGF was effective modality in the treatment of disc displacement with reduction (DDWR) of tempromandibular joint with minimal postoperative complication after failure of conservative treatment. Arthrocentesis was safe and also minimally invasive method in the treatment of tempromandibular disorders. Efficacy of CGF injection was better than arthrocentesis in the treatment of pain and limited mouth opening.

\section{REFERENCES}

1. DuBrul, EL. Sicher's oral anatomy. 7th ed. St. Louis (MO): C.V. Mosby; press, 1980: 146-61.

2. Cooper BC, Kleinberg I. Establishment of a temporomandibular physiological state with neuromuscular orthosis treatment affects reduction of TMD symptoms in 313 patients. Cranio 2008; 26: 104-17. 
3. Manfredini, D, Marini M, Pavan C, Pavan L, Guarda-Nardini L. Psychosocial profiles of painful TMD patients. J Oral Rehabil 2009; 36:193-8.

4. Burnouf T, Goubran HA, Chen TM, El-Ekiaby M, Radosevic. Blood-derived biomaterials and platelet growth factors in regenerative medicine. Blood Reviews 2013; 27:77-89.

5. Bianco P, Robey PG. Stem cells in tissue engineering. Nature.2001;414:118-21.

6. Pihut M, Szuta M, Ferendiuk E, Zeńczak, Więckiewicz D. Evaluation of Pain Regression in Patients with Temporomandibular Dysfunction Treated by Intra- Articular Platelet-Rich Plasma Injections: A Preliminary Report. Biomed Res Int 2014; 2014:132.

7. Machoň V, Ǩehořová M, Šedý J, Foltán R. Platelet-Rich Plasma in Temporomandibular Joint Osteoarthritis Therapy: A 3-Month Follow-Up Pilot Study. J Arthritis 2013; 2: 112 .

8. Yu B, Wang Z. Effect of concentrated growth factors on beagle periodontal ligament stem cells in vitro. Mol. Med. Rep.2014; 9: 235-242.

9. Murakami K, Hosaka H, Moriya Y, Segami N, Iizuka T. Short-term treatment outcome study for the management of temporomandibular joint closed lock: a comparison of arthrocentesis to nonsurgical therapy and arthroscopic lysis and lavage. Oral Surg Oral Med Oral Pathol Oral Radiol Endodontol 1995; 80: 253-7.

10. Rodella LF, Favero G, Boninsegna R, et al. Growth factors, CD34 positive cells, and fibrin network analysis in concentrated growth factors fraction. Microsc Res Tech 2011;74:772-7.

11. Akeda K, An H, Okuma M, Attawia M, Miyamoto K, Thonar E, et al. Platelet-rich plasma stimulates porcine articular chondrocyte proliferation. Osteoarthritis and Cartilage Journal 2006; 14:98-80.

12. Botelho A, De Arruda Veiga M. Influence of sex on temporomandibular disorder pain: a review of occurrence and development. Braz J Oral Sci 2008; 7: 1631-5.

13. Schär MO, Diaz-Romero J, Kohl S, Zumstein MA, Nesic D. Platelet-rich concentrates differentially release growth factors and induce cell migration in vitro. Clin Orthop Relat Res. 2015; 473: 1635-1643.

14. Gassling VL, Acil Y, Springer IN, Hubert N, Wiltfang J. Platelet-rich plasma and platelet-rich fibrin in human cell culture. Oral Surg Oral Med Oral Pathol Oral Radiol Endod. 2009; 108: 48-55.

15. Passaretti F, Tia M, D’Esposito V, De Pascale M, Del Corso M, Sepulveres R, et al. Growth-promoting action and growth factor release by different platelet derivatives. Platelets. 2014; 25:252.

16. Giacomello M, Giacomello A, Mortellaro C, Gallesio G, Mozzati M. Temporomandibular joint disorders treated with articular injection: the effectiveness of plasma rich in growth factors Endoret. J Craniofac Surg 2015; 26: 709-685.

17. Nitzan DW, Dolwick MF, Martinez GA. Temporomandibular joint arthrocentesis: a simplified treatment for severe, limited mouth opening. J Oral Maxillofac Surg. 1991; 49: 1163-7.

18. Moon S, Lee S, Ryu J. Ultrasound-guided Platelet-rich Plasma Prolotherapy for Temporomandibular Disorders. J Oral Med Pain 2014; 39: 140-5.

19. Yoda T, Imai H, Shinjyo Y, Sakamoto I, Abe M, Enomoto $\mathrm{S}$. Effect of arthrocentesis on TMJ disturbance of mouth closure with loud clicking: a preliminary study. J Craniomandibular Pract 2002; 20: 18-22. 